\title{
Form field theory on Calabi-Yau three-fold
}

\author{
Farhang Loran and Hesam Soltanpanahi \\ Department of Physics, Isfahan University of Technology, \\ Isfahan 84156-83111, Iran \\ loran@cc.iut.ac.ir, h_soltanpanahi@ph.iut.ac.ir
}

\begin{abstract}
We show how the natural Abelian duality of 2 and 3-form gravity theories on seven-dimensional manifold $C Y_{3} \times S^{1}$ leads to an S-duality between 2 and 3 -form theories on simply connected $C Y_{3}$. The massless sector of the 2-form field theory on $C Y_{3}$ corresponds to the A model string field theory. We discuss the complex structure independence of the 2-form theory for a general Kähler manifold and derive the holomorphic anomaly equations for simply connected $C Y_{3}$.
\end{abstract}

Contents

1 Introduction and conclusion

2 Abelian duality in seven dimensions

2.1 Abelian duality

2.2 Seven-dimensional Abelian duality 


\subsection{Equation of motion and gauge fixing}

3 Reduction to Calabi-Yau three-fold

4 Canonical quantization and position/momentum duality

\section{$4.1 \quad$ S-duality on $\mathrm{CY}_{3}$}

653

5 Theory of Kähler gravity

6 Quantum background independence

6.1 The structure of connections

658

6.2 Complex structure independence in form field theories

A Abelian duality in seven dimensions 661

B Relation between the operators $d$ and $*$ in $m$ and $m+1$ dimensions

\section{References}

665

\section{Introduction and conclusion}

Since the beginning of the 1990s, form theories of gravity have been studied by several authors. Interesting examples of them, related to the present work are the Chern-Simons theory exposition of three-dimensional gravity [1], the description of the target space theory of A model topological string in terms of variations of the Kähler 2-form [2], and the description of the target space of B model topological string theory in terms of variations of the holomorphic 3 -form [3-9].

In [10] Vafa conjectured that there is a mirror symmetry between topological A and B string theories. For topological string theories targeting a simply connected Calabi-Yau three-fold, the observables of the A model are in one-to-one correspondence with the elements of $H^{1,1}\left(C Y_{3}\right)$ while they are independent of the complex structure moduli. On the other hand, for the topological B model the observables are in one-to-one correspondence with 
the elements of $H^{2,1}\left(\mathrm{CY}_{3}\right)$ but independent of the Kähler structure. The existence of such theories is supported by the Candelas observation in [11] that the moduli space of $C Y_{3}$ factorizes into a direct product of complex structure moduli and Kähler moduli at least locally. For a simply connected Calabi-Yau three-fold, $h^{1,1}$ and $h^{2,1}$ i.e., the dimensions of $H^{1,1}$ and $H^{2,1}$, respectively, are the only non-trivial independent Hodge numbers. A Calabi-Yau three-fold mirror to a given Calabi-Yau three-fold is identified with Hodge numbers,

$$
\tilde{h}^{1,1}=h^{2,1}, \quad \tilde{h}^{2,1}=h^{1,1} .
$$

The conjectured mirror symmetry of [10] is a duality between the A model on a $C Y_{3}$ and the $\mathrm{B}$ model on the corresponding mirror, which has been confirmed for example for quintics and the corresponding mirrors. See, e.g., [12] and references therein.

Roughly speaking the relation between the A model and B model topological string theories resembles the relation between type IIA and IIB superstring theories. The T-duality between type IIA and IIB superstring theories is explained in terms of an M-theory defined on the superstring target space trivially fibred over an extra Euclidean $S^{1}$. Therefore it is natural to expect that there exists a topological M-theory in seven dimensions, which can be used to describe the duality of topological string field theories. Also, one expects that this seven-dimensional theory is defined on $C Y_{3} \times S^{1}$, where $C Y_{3}$ is trivially fibred over $S^{1}$. The trivially fibred $C Y_{3} \times S^{1}$ is one of the simplest examples of $G_{2}$ manifolds, which are the natural classes of sevendimensional manifolds preserving some supersymmetry and are purely geometric [13]. This is the setup of [14], where a duality of A and B model string field theories on the same target space is studied. In principle, the mirror symmetry of the A and B string field theories can be given by a topological M-theory for which the $C Y_{3}$ is non-trivially fibred over $S^{1} / \mathbb{Z}_{2}$ whose fixed points are the locations of a Calabi-Yau three-fold and the corresponding mirror.

The topological A and B string field theories can be considered as form gravity theories in six or seven dimensions. Recently, in $[15,16]$ it is conjectured that there is an S-duality between A and B models targeting the same $C Y_{3}$. They have shown that the perturbative amplitudes of the $\mathrm{B}$ model capture the non-perturbative amplitudes of the A model and vice versa. In [14] it is demonstrated that there is a seven-dimensional form theory on $C Y_{3} \times S^{1}$, called topological M-theory, which is related to both 2 and 3-form theories on the same $C Y_{3}$, and at least at the classical level these form theories are related to the topological A and B models, respectively. Also, they have deduced a position/momentum duality, called S-duality, between the 
the 2 and 3-form theories. They have explained that the S-duality, indeed, is a duality of $\mathrm{A}$ and $\mathrm{B}+\overline{\mathrm{B}}$ models. An evidence for that observation is provided by the BPS counting of black hole states [14]. In the form field theories which we are going to discuss in the present work, one encounters a similar duality of $\mathrm{A}$ and $\mathrm{B}+\overline{\mathrm{B}}$ models but in the case of non-simply connected Calabi-Yau three-folds.

The seven-dimensional manifolds have a specific property which makes them useful to be used in a description of the A and B models in terms of an M-theory. From Abelian duality we know that on every $m$-dimensional manifold $M$ there is a duality between $r$-forms and $(m-r-2)$-forms [17]. Therefore on a seven-dimensional manifold there is a natural Abelian duality between 2 and 3-form field theories, while in six dimensions there does not exist an Abelian duality between 2 and 3-form fields. In this paper we consider the Abelian duality between 2 and 3-form field theories on a trivially fibred $C Y_{3} \times S^{1}$. By applying the usual dimensional reduction method we obtain 2- and 3-form field theories on $C Y_{3}$ which are dual to each other in the sense of [14].

The physical quantities of the form field theories we consider in this paper are by construction elements of $H^{1,1}\left(C Y_{3}\right)$, since we are considering a simply connected Calabi-Yau three-fold $\left(h^{1,0}=h^{2,0}=0\right)$, and $H^{3}\left(C Y_{3}\right)=H^{3,0} \oplus$ $H^{2,1} \oplus H^{1,2} \oplus H^{0,3}$. The theory by construction is independent of the complex structure. But as can be seen from the foregoing Hodge decomposition any representation of the observables amounts to defining a complex structure. Therefore, the physical observables satisfy a set of identities imposed by background independence conditions that ensure the independence of the complex structure $[1,6,9]$. For the 2-form theory, background independence is reasonable if the 2-form field theory is going to be interpreted as the A model string field theory. As we will show the massless sector of the 2-form theory we obtain by dimensional reduction is the theory of Kähler deformation of the Calabi-Yau three-fold. To our knowledge such a theory has been proposed for the first time in [2] where a theory of Kähler deformation was constructed in analogy to the Kodaira-Spencer theory of complex structure deformation, following the mirror symmetry. We show that our proposal for the 2-form theory coincides with the AKS theory of [2] if all terms in the perturbation theory are considered. The 3-form theory studied here is not related to the KS theory since it is the Abelian dual and not the mirror of the 2-form theory and is defined on the same Calabi-Yau three-fold and not on its mirror three-fold. We further explain this point in terms of a classic/quantum correspondence between the two form field theories.

In this paper we study duality of 2 and 3-form theories that at the classical level correspond to the topological A and B string field theories on the same 
$\mathrm{CY}_{3}$ respectively. To this aim we use Abelian duality in seven dimensions. We explicitly show that the Abelian duality of the 2 and 3-form gravity theories on a trivially fibred $C Y_{3} \times S^{1}$ gives, after Kaluza-Klein reduction, an S-duality between 2 and 3-form gravity theories on $C Y_{3}$. From a sevendimensional point of view the Abelian duality gives the position/momentum duality of [14]. Furthermore both in six and seven dimensions the Abelian duality implies a classic/quantum correspondence between the two theories. In fact, the classical regime of the 2-form theory corresponds to the 3 -form theory in the $\hbar \rightarrow \infty$ limit. In the classical limit, the 2 -form theory reduces to its zero mode sector, which is the theory of the Kähler structure deformation. The other extreme is the classical regime of the 3-form field theory, which corresponds to the $\hbar \rightarrow \infty$ limit of the 2 -form theory. The 3 -form theory in this limit reduces to its zero mode sector in which the physical quantities are the elements of $\mathrm{H}^{3}\left(\mathrm{CY}_{3}\right)$. It is tempting to anticipate a connection between this theory and the topological B string field theory. But, we notify again that, the 3-form theory should not be expected to be the KodairaSpencer theory since one is considering a trivially fibred $C Y_{3}$ over $S^{1}$.

In a proper gauge we quantize these form gravity theories and describe the Abelian duality as a map between the corresponding annihilation and creation operators. An interesting feature of these theories is the existence of a one parameter family of vacua,

$$
\mid \text { vac. }\rangle_{\theta}=\mathrm{e}^{\mathrm{i} \theta a_{0}}|0\rangle,
$$

where $a_{0}$ corresponds to the massless modes. The quantum theory of 2-form field theory should be further equipped by suitable holomorphic anomaly equations ensuring the background independence since this is a gravity theory for the A model. On the dual side, quantum background independence for 3 -form fields is previously worked out in $[6,7,9]$.

The plan of this paper is as follows: in Section 2, after a brief review of Abelian duality on general $m$-dimensional manifolds we study Abelian duality in the case of the seven-dimensional manifold $C Y_{3} \times S^{1}$ between 2 and 3 -form theories. In a proper gauge the equations of motion of the original field theory reduce to the equations for components of fields along $C Y_{3}$. This makes the dimensional reduction of seven-dimensional form field theories to six dimensions straightforward. We show that classically the Abelian duality is a position/momentum duality. In the path integral formulation of the Abelian duality we show that it describes a classic/quantum correspondence between the two form field theories. In Section 3, we derive 2 and 3-form gravity theories on $C Y_{3}$ by applying the KK-dimensional reduction method to the original seven-dimensional form field theories and reinterpret the classic/quantum correspondence in six dimensions. Canonical 
quantization of the seven-dimensional field theories is studied in Section 4. Section 5 is devoted to the connection between the 2-form theory and the theory of Kähler gravity. In Section 6 we first construct a method to study the complex structure deformation of a complex manifold. Then we study the quantum background independence of the massless sector of the 2-form theory. In Appendix A, we derive equations used in Section 2. The mathematical background of Section 3 is provided in Appendix B.

\section{Abelian duality in seven dimensions}

In this section we derive Abelian duality between 2 and 3-forms in seven dimensions. For doing this in Subsection 2.1 we study Abelian duality of $r$ and $(m-r-2)$-forms on an $m$-dimensional manifold $M$. Then in Subsection 2.2 we use Abelian duality in the seven-dimensional manifold to explain relation between 2 and 3 -form theories in seven dimensions. In Subsection 2.3, for the special seven-dimensional manifold $C Y_{3} \times S^{1}$ by using gauge fixing condition and after decomposing the components of 2 and 3forms along $\mathrm{CY}_{3}$ and $S^{1}$ we obtain equations of motion for components of forms along $\mathrm{CY}_{3}$ and the classical duality between those components. The result is a duality between the 2 and 3 -form field theories in six dimensions, which is the inheritance of the seven-dimensional Abelian duality.

\subsection{Abelian duality}

In this subsection we review Abelian duality in $m$-dimensional Riemannian manifolds $M$ and define form field theories on such manifolds. All of the operators that one encounters in our form theories are shown in the following diagram [17]:

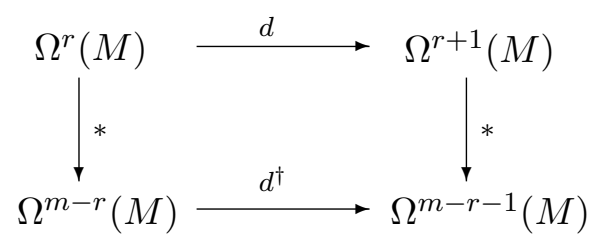

where $\Omega^{r}(M)$ is the set of $r$-forms on $M$. If $M$ is a Riemannian manifold, a general $r$-form $\eta_{r}$ satisfies the following equations:

$$
\begin{aligned}
d^{\dagger} \eta_{r} & =(-)^{m(r+1)+1} * d * \eta_{r}, \\
* * \eta_{r} & =(-)^{r(m-r)} \eta_{r},
\end{aligned}
$$


where $d$ is the exterior derivative operator and $*$ is the Hodge star operator. There are the same relations with an extra $(-1)$ for Lorentzian manifolds. One can define an inner product of two $r$-forms $\eta_{r}$ and $\zeta_{r}$ on a manifold $M$,

$$
\left(\eta_{r}, \zeta_{r}\right)=\int_{M} \eta_{r} \wedge * \zeta_{r},
$$

which is positive definite for Riemannian manifold, $\left(\varpi_{r}, \varpi_{r}\right) \geq 0$, and the equality is satisfied if and only if $\varpi_{r}=0$. Furthermore, $\left(\eta_{r}, \zeta_{r}\right)=\left(\zeta_{r}, \eta_{r}\right)$ [18].

For a closed $r$-form $\eta_{r}$ which obeys the condition $d \eta_{r}=0$ one can locally assume that $\eta_{r}=d \zeta_{r-1}$. This follows from the fact that the operator $d$ is nilpotent namely $d^{2}=0$. Also for the nilpotent operator $d^{\dagger}$ if $d^{\dagger} \eta_{r}=0$ then locally $\eta_{r}=d^{\dagger} \zeta_{r+1}$. Therefore if

$$
d^{\dagger}\left(d \gamma_{r}\right)=(-)^{m(r+2)+1} * d * d \gamma_{r}=0,
$$

in which we have used equation (2.1), then by using equation (2.2), one obtains

$$
d\left(* d \gamma_{r}\right)=0 .
$$

Thus there exists an $(m-r-2)$-form $\omega_{m-r-2}$ for which

$$
* d \gamma_{r}=d \omega_{m-r-2} .
$$

This relation is known as Abelian duality. Considering both the $r$-from $\gamma_{r}$ and the $(m-r-2)$-form $\omega_{m-r-2}$ as classical fields of two different form field theories, then equation (2.6) is a duality of the corresponding equations of motions.

By using a positive-definite inner product we can construct an action for any $r$-form $\eta_{r}$ such that it gives an equation of motion for $\eta_{r}$ similar to equation (2.4). This action is defined in the following way:

$$
S(\eta) \sim\left(d \eta_{r}, d \eta_{r}\right)=\int_{M} d \eta_{r} \wedge * d \eta_{r},
$$

for which, using the identity,

$$
\left(d \alpha_{r}, \beta_{r}\right)=\left(\alpha_{r}, d^{\dagger} \beta_{r}\right),
$$

the equation of motion is given by

$$
d^{\dagger} d \eta_{r}=0 .
$$


Also, it is obvious that this action is invariant under the following gauge transformation:

$$
\eta_{r} \rightarrow \eta_{r}+d \theta_{r-1}
$$

where $\theta_{r-1} \in \Omega^{r-1}(M)$. The gauge freedom can be fixed by using the following gauge condition:

$$
d^{\dagger} \eta_{r}=0 .
$$

By using both equation of motion (2.9) and gauge fixing condition (2.11) one obtains that ${ }^{1}$

$$
\Delta \eta_{r}=0
$$

Equation (2.12) implies that the gauge (2.11) does not fix the gauge freedom completely since one can still add an arbitrary harmonic form to $\eta$.

\subsection{Seven-dimensional Abelian duality}

Now, we are ready to study Abelian duality of 2 and 3-form field theories on $\Sigma=C Y_{3} \times S^{1}$. Considering $C \in \Omega^{3}(\Sigma)$ and $K \in \Omega^{2}(\Sigma)$, we assume that both of these fields obey the following equations of motion: ${ }^{2}$

$$
d_{7}^{\dagger} d_{7} C=0, \quad d_{7}^{\dagger} d_{7} K=0,
$$

with the Abelian duality relation

$$
*_{7} d_{7} C=d_{7} K .
$$

To study the quantum version of this classical duality one needs to compare the partition functions of both theories and consequently one should define an action functional for each of them. As we saw in the last subsection, on a compact orientable Riemannian manifold one can define a positive-definite action for the 3 -form $C$ :

$$
S(C)=\frac{\Lambda}{4 \pi}\left(d_{7} C, d_{7} C\right)=\frac{\Lambda}{4 \pi} \int_{\Sigma} d_{7} C \wedge *_{7} d_{7} C,
$$

which gives equation (2.13) for the 3-form $C$. The partition function of theory is

$$
Z=\int \mathcal{D} \phi \mathrm{e}^{-S(\phi)}
$$

To obtain the Abelian duality, following [19], we introduce new variables such that if we integrate them out from the partition function we get back

\footnotetext{
${ }^{1}$ By definition on $m$-dimensional manifold $\Delta=d^{\dagger} d+d^{\dagger} d$.

${ }^{2}$ Subscript of operators $d$ and $*$ refers to the dimensions of manifolds on which the operators are defined.
} 
to the original theory, and if we integrate out the old variables instead of the new ones it produces the dual theory. To this aim we interpret $C$ as a section of a trivial $S^{1}$-bundle $\mathcal{L}_{A}$ with a connection $A$, and write an action which includes the covariant derivative $D_{7 A} C=d_{7} C+A$ in the following way:

$$
S(C, A)=\frac{\Lambda}{4 \pi} \int_{\Sigma} D_{7 A} C \wedge *_{7} D_{7 A} C .
$$

There is a simple mechanism for recovering the old action. We introduce a third field $K$ which plays the role of the Lagrange multiplier. We take $K$ to be a section from $\Sigma$ to $S^{1}$, and write an action

$$
S(C, A, K)=\frac{\Lambda}{4 \pi} \int_{7} D_{7 A} C \wedge *_{7} D_{7 A} C-\frac{i}{2 \pi} \int_{7} K \wedge d_{7} A .
$$

This action is invariant under the gauge transformation

$$
\begin{aligned}
& A \rightarrow A+d B, \\
& C \rightarrow C-B,
\end{aligned}
$$

where $\mathrm{B}$ is a 3 -form. To see that $S(C, A, K)$ is equivalent to $S(C)$, one can note that the equation of motion for $K$ is $d_{7} A=0$. By imposing this condition and using the $A=0$ gauge the original theory gets recovered.

The above naive argument can be made exact as follows. Consider the partition function of the whole theory,

$$
Z=\frac{1}{\operatorname{vol}(G)} \int \mathcal{D} C \mathcal{D} A \mathcal{D} K \exp \left[-\frac{\Lambda}{4 \pi} \int_{7} D_{7 A} C \wedge *_{7} D_{7 A} C+\frac{i}{2 \pi} \int_{7} K \wedge d_{7} A\right],
$$

in which $G$ is the gauge group for $\mathcal{L}_{A}$. By using the delta function,

$$
\int \frac{d x}{2 \pi} \mathrm{e}^{\mathrm{i} x y}=\delta(y)
$$

the $K$ integral sets $d A$ equal to zero. But we are studying circle-valued functions and we should treat this statement carefully. In general, $K=$ $K_{\text {har }}+K_{S^{1}}$, where $K_{\text {har }}: \Sigma \rightarrow S^{1}$ is a circle valued 2-form such that $d K_{\text {har }}$ is a harmonic 3-form representative in the cohomology class of $d K$, and $K_{S^{1}}$ is a single valued real function on $S^{1}$. Introducing a basis for harmonic 3forms $\lambda_{j}$, where $j=1, \ldots, h^{3}(\Sigma),{ }^{3}$ one can write $d K_{\text {har }}=\sum_{j=1}^{h^{3}(\Sigma)} 2 \pi m_{j} \lambda_{j}$,

$$
{ }^{3} h^{3}(\Sigma)=2+2 h^{2,1}\left(C Y_{3}\right)+h^{1,1}\left(C Y_{3}\right)[18]
$$


in which $m_{j} \in \mathbb{Z}$. Now one obtains

$$
\begin{aligned}
\int & \mathcal{D} K \exp \left[\frac{\mathrm{i}}{2 \pi} \int_{\Sigma} K \wedge d_{7} A\right] \\
& =\int \mathcal{D} K_{S^{1}} \exp \left[\frac{\mathrm{i}}{2 \pi} \int_{\Sigma} K_{S^{1}} \wedge d_{7} A\right] \times \sum_{d K_{\mathrm{har}} \in H^{3}(\Sigma, 2 \pi \mathbb{Z})} \mathrm{e}^{-\frac{\mathrm{i}}{2 \pi} d K_{\mathrm{har}} \wedge A} \\
& =\delta\left(d_{7} A\right) \prod_{j}\left(\sum_{m_{j} \in \mathbb{Z}} \mathrm{e}^{-\mathrm{i} m_{j} \int_{\Sigma} \lambda_{j} \wedge A}\right) .
\end{aligned}
$$

The first term shows that $A$ is a flat connection, and among flat connections the gauge equivalence classes $[A]$ are labelled by holonomies, or equivalently by the quantities $\int_{\Sigma} \lambda_{j} \wedge A$. The remaining part of the integral is gauge invariant therefore one can fix the gauge and integrate over the space of gauge equivalent classes and obtain (omitting the factor $(\operatorname{vol}(G))^{-1}$ )

$$
\begin{aligned}
Z & =\int \mathcal{D} C \mathcal{D}[A] \mathrm{e}^{-\frac{\Lambda}{4 \pi} \int_{\Sigma} D_{7 A} C \wedge_{*_{7}} D_{7 A} C} \delta\left(d_{7} A\right) \prod_{j}\left(\sum_{m_{j} \in \mathbb{Z}} \mathrm{e}^{-\mathrm{i} m_{j} \int_{\Sigma} \lambda_{j} \wedge A}\right) \\
& =\int \mathcal{D} C \mathrm{e}^{-\frac{\Lambda}{4 \pi} \int_{\Sigma} D_{7 A} C \wedge_{*_{7}} D_{7 A} C} \delta\left(d_{7} A\right) \delta\left(\int_{\Sigma} \lambda_{j} \wedge A=0 \bmod 2 \pi \mathbb{Z}\right) \\
& =\int \mathcal{D} C \mathrm{e}^{-\frac{\Lambda}{4 \pi} \int_{\Sigma} d_{7} C \wedge_{7} d_{7} C} .
\end{aligned}
$$

In the last equality we have used the fact that the delta functions sets the holonomies equal to zero as well as curvature. Thus $A$ is zero modulo gauge transformations, and the new theory is indeed equivalent to the original theory.

Now we want to integrate out $C$ and $A$ to obtain a new theory in terms of the 2-form field $K$. First, we integrate out $C$ by using the Faddeev-Popov method and then integrate $A$ out by the Gausian method. For the first step, we fix the gauge freedom (2.19) using the gauge $C=0$. In this gauge the path integral (2.20) reduces to

$$
\int \mathcal{D} A \mathcal{D} K \mathcal{D} \bar{c} \mathcal{D} c \exp \left[-\frac{\Lambda}{4 \pi} \int_{\Sigma} A \wedge *_{7} A+\frac{\mathrm{i}}{2 \pi} \int_{\Sigma} K \wedge d_{7} A-\frac{\Lambda}{4 \pi} \int_{\Sigma} \bar{c} 1 c\right]
$$

The last term arises from gauge fixing by the Faddeev-Popov method, and $c$ and $\bar{c}$ are any allowed grassmanian forms on $\Sigma$. 
For integrating out $A$, we complete the square. To do this, we introduce new variable $A^{\prime}=A+\frac{i}{\Lambda} *_{7} d_{7} K$. Then we have

$$
\left(\int \mathcal{D} K \mathrm{e}^{-\frac{1}{4 \pi \Lambda} \int_{\Sigma} d K \wedge_{7} d_{7} K}\right)\left(\int \mathcal{D} A^{\prime} \mathrm{e}^{-\frac{\Lambda}{4 \pi} \int_{\Sigma} A^{\prime} \wedge_{7} A^{\prime}}\right)\left(\int \mathcal{D} \bar{c} \mathcal{D} c \mathrm{e}^{-\frac{\Lambda}{4 \pi} \int_{\Sigma} \bar{c} 1 c}\right) .
$$

The first term is the partition function of the dual theory which is the 2 -form theory in seven dimensions. The last two terms give a topological factor which depends on the number of independent harmonic forms in seven-dimensional manifold $C Y_{3} \times S^{1}$ (see Appendix A for this subtlety). Consequently, we have the following equality between the partition functions of two form theories:

$$
\int \mathcal{D} C \mathrm{e}^{-\frac{\Lambda}{4 \pi} \int_{\Sigma} d C \wedge_{*} *_{7} C}=\mathcal{C}(\Sigma) \int \mathcal{D} K \mathrm{e}^{-\frac{1}{4 \pi \Lambda} \int_{\Sigma} d K \wedge^{*} d_{7} K} .
$$

It is notable that the coupling $\Lambda$ in the first theory is mapped to $1 / \Lambda$ in the second theory. Therefore there is a classic/quantum correspondence between these form theories. Equation (2.26) is given in the natural units $(c=\hbar=1)$. Recovering $\hbar$ in equation $(2.26)$ one obtains $Z(\phi)=\int \mathcal{D} \phi \mathrm{e}^{-\frac{1}{\hbar} S(\phi)}$. Thus, in 3 -form (2-from) theory $1 / \Lambda(\Lambda)$ acts as $\hbar$ and we can study classical and quantum regimes of each theory in the limit of $\Lambda$ tending to 0 or $\infty$.

\subsection{Equation of motion and gauge fixing}

The equation of motion for 3-form $C$ given by the action (2.15) is

$$
d_{7}^{\dagger} d_{7} C=0 .
$$

After the decomposition $C=\gamma+d t \wedge \alpha$ where $\gamma$ is a 3 -form along $C Y_{3}$ and $d t \wedge \alpha$ is a 1 -form along $S^{1}$ and a 2 -form along $C Y_{3}$, and also the decomposition of the operator $d_{7}$ into its components along $C Y_{3}$ and $S^{1}$, $d_{7}=d_{C Y}+d t \wedge d / d t$, the equation of motion (2.27) reduces to ${ }^{4}$

$$
\begin{array}{r}
d_{C Y}^{\dagger} d_{C Y} \gamma-\ddot{\gamma}-d_{C Y} \dot{\alpha}=0, \\
d_{C Y}^{\dagger} d_{C Y} \alpha+d_{C Y}^{\dagger} \dot{\gamma}=0,
\end{array}
$$

where dot "." stands for a derivation with respect to $t$ which parameterizes the $S^{1}$. To obtain this results one needs the relations between operators $d$

\footnotetext{
${ }^{4}$ We can obtain these equations of motion by putting decomposition of $C$ in action (2.15) and using Euler-Lagrange equation.
} 
and $*$ in the seven-dimensional manifold $\Sigma$ and the six-dimensional manifold $C Y_{3}$ which can be found in Appendix B.

The action (2.15) is invariant under the gauge transformation $C \rightarrow C+$ $d_{7} \rho$, where $\rho$ is a 2 -form along $\Sigma$. We can fix this gauge freedom by $d_{7}^{\dagger} C=0$ gauge. In terms of $\gamma$ and $\alpha$, the gauge transformation is

$$
\gamma \rightarrow \gamma+d \rho_{1}, \quad \alpha \rightarrow \alpha+\dot{\rho}_{1}+d \rho_{2},
$$

in which $\rho=\rho_{1}+\rho_{2} \wedge d t$ and the gauge fixing condition is equivalent to

$$
\begin{array}{r}
d_{C Y}^{\dagger} \gamma+\dot{\alpha}=0, \\
d_{C Y}^{\dagger} \alpha=0 .
\end{array}
$$

By operating $d_{C Y}$ and $d / d t$ on the first equation and inserting the result into the equations of motion $(2.28,2.29)$, one obtains

$$
\begin{aligned}
\Delta_{C Y} \gamma-\ddot{\gamma} & =0, \\
\Delta_{C Y} \alpha-\ddot{\alpha} & =0 .
\end{aligned}
$$

Similar calculations can be done in the case of the 2-form theory in seven dimensions. From equation (2.26) one verifies that the action for $K$ is

$$
S(K)=\frac{1}{4 \pi \Lambda} \int_{\Sigma} d_{7} K \wedge *_{7} d_{7} K .
$$

After the decomposition $K=\omega+d t \wedge \beta$, where $\omega$ and $\beta$ are 2-form and 1form along $C Y_{3}$ respectively, the equation of motion for $K$, i.e., $d_{7}^{\dagger} d_{7} K=0$, reduces to

$$
\begin{aligned}
d_{C Y}^{\dagger} d_{C Y} \omega-\ddot{\omega}+d_{C Y} \dot{\beta} & =0 \\
d_{C Y}^{\dagger} d_{C Y} \beta-d_{C Y}^{\dagger} \dot{\omega} & =0 .
\end{aligned}
$$

The gauge symmetry of the action for 2 -form $K$ is

$$
K \rightarrow K+d \theta
$$

where $\theta$ is an 1 -form along $\Sigma$. The gauge fixing condition $d_{7}^{\dagger} K=0$ gives

$$
\begin{array}{r}
d_{C Y}^{\dagger} \omega-\dot{\beta}=0, \\
d_{C Y}^{\dagger} \beta=0,
\end{array}
$$


which can be used to simplify the equations of motion $(2.36,2.37)$ as follows:

$$
\begin{aligned}
& \Delta_{C Y} \omega-\ddot{\omega}=0, \\
& \Delta_{C Y} \beta-\ddot{\beta}=0 .
\end{aligned}
$$

The classical duality (2.14) between the 3 -form $C$ and 2 -form $K$ results in the following duality relations:

$$
\begin{aligned}
& *_{C Y} d_{C Y} \gamma=\dot{\omega}+d_{C Y} \beta, \\
& *_{C Y}\left(\dot{\gamma}+d_{C Y} \alpha\right)=d_{C Y} \omega .
\end{aligned}
$$

In the next sections we will argue that these relations give us an interpretation of the Abelian duality as the position/momentum duality [14].

The 3 -form action (2.15) in terms of the $\gamma$ and $\alpha$ components is, ${ }^{5}$

$$
S(\gamma, \alpha)=\frac{\Lambda}{4 \pi} \int_{S^{1}} d t\{(\dot{\gamma}, \dot{\gamma})+(d \gamma, d \gamma)+2(\dot{\gamma}, d \alpha)+(d \alpha, d \alpha)\}
$$

Since we are interested in form field theories on $C Y_{3}$, we use the gauge freedom (2.30) to set

$$
\alpha=0 .
$$

In this gauge the action (2.45) simplifies to

$$
S(\gamma)=\frac{\Lambda}{4 \pi} \int_{S^{1}} d t\{(\dot{\gamma}, \dot{\gamma})+(d \gamma, d \gamma)\}
$$

One should note that there is still a gauge freedom

$$
\gamma \rightarrow \gamma+d \tilde{\rho}_{1}
$$

where $\tilde{\rho}_{1}$ is an arbitrary 2 -form independent of $t$

$$
\tilde{\rho}_{1} \in \Omega^{2}\left(C Y_{3}\right) .
$$

By the same argument one can get rid of the 1-form $\beta$ in the 2 -from theory to obtain $S(\omega)=\frac{1}{4 \pi \Lambda} \int_{S^{1}} d t\{(\dot{\omega}, \dot{\omega})+(d \omega, d \omega)\}$. In these gauges the classical dualities (2.43) and (2.44) reduce to

$$
\begin{aligned}
& * d \gamma=\dot{\omega}, \\
& * \dot{\gamma}=d \omega .
\end{aligned}
$$

\footnotetext{
${ }^{5}$ Henceforth, all operators and the corresponding inner products are defined on $C Y_{3}$ and we omit the subscript $C Y$.
} 


\section{Reduction to Calabi-Yau three-fold}

In this section we study the reduction of 2 and 3 -form theories in seven dimensions to form gravity theories on six-dimensional manifold $C Y_{3}$ by using Kaluza-Klein method. We assume that $R$ is the radius of $S^{1}$ over which the $C_{3}$ is fibred trivially. In this way we can expand all forms:

$$
\begin{aligned}
\gamma & =\sum_{n \in \mathbb{Z}} \gamma_{n} \mathrm{e}^{\mathrm{i} 2 \pi n t / R}, \\
\omega & =\sum_{n \in \mathbb{Z}} \omega_{n} \mathrm{e}^{\mathrm{i} 2 \pi n t / R},
\end{aligned}
$$

where $\gamma_{n}$ and $\omega_{n}$ are defined on $C Y_{3}$ and their dependence on the extra dimension $t$ along $S^{1}$ is given in the phase $\mathrm{e}^{\mathrm{i} 2 \pi n t / R}$. Thus both actions on the $C Y_{3}$ reduce to

$$
\begin{aligned}
& S\left(\gamma_{n}\right)=\frac{\Lambda}{4 \pi} \sum_{n \in \mathbb{Z}}\left\{\frac{4 \pi^{2} n^{2}}{R^{2}}\left(\gamma_{n}, \gamma_{-n}\right)+\left(d \gamma_{n}, d \gamma_{-n}\right)\right\}, \\
& S\left(\omega_{n}\right)=\frac{1}{4 \pi \Lambda} \sum_{n \in \mathbb{Z}}\left\{\frac{4 \pi^{2} n^{2}}{R^{2}}\left(\omega_{n}, \omega_{-n}\right)+\left(d \omega_{n}, d \omega_{-n}\right)\right\} .
\end{aligned}
$$

To obtain the above results the following identity is used:

$$
\int_{S^{1}} d t \mathrm{e}^{\mathrm{i} 2 \pi(m+n) t / R}=\delta_{m+n, 0}
$$

In addition, all of the equations of motion on $\mathrm{CY}_{3}$ in terms of $\mathrm{KK}$ modes simplify to

$$
\begin{aligned}
d^{\dagger} d \gamma_{n} & =-\frac{4 \pi^{2} n^{2}}{R^{2}} \gamma_{n}, \\
d^{\dagger} d \omega_{n} & =-\frac{4 \pi^{2} n^{2}}{R^{2}} \omega_{n} .
\end{aligned}
$$

We see that coefficient the $\frac{4 \pi^{2} n^{2}}{R^{2}}$ in both theories gives the KK mass squared of the $n$th mode.

There is a subtlety in the partition functions of these theories as can be seen from equations (3.3) and (3.4). In the classical limit of the 3 -form (2form) theory $\Lambda \rightarrow \infty(1 / \Lambda \rightarrow \infty)$, which corresponds to $\hbar \rightarrow 0$, the partition function gets localized around the classical trajectory. By using equations 
of motion (3.6), (3.7) one can obtain the following inner product for the KK modes on the Calabi-Yau three-fold:

$$
\begin{aligned}
\left(d \gamma_{n}, d \gamma_{n}\right) & =-\frac{4 \pi^{2} n^{2}}{R^{2}}\left(\gamma_{n}, \gamma_{n}\right), \\
\left(d \omega_{n}, d \omega_{n}\right) & =-\frac{4 \pi^{2} n^{2}}{R^{2}}\left(\omega_{n}, \omega_{n}\right) .
\end{aligned}
$$

Recalling that for Riemannian manifolds the inner product is positive definite, one verifies that the solution of the equations of motion is $\gamma_{n}=0=\omega_{n}$ for $n \neq 0$. Consequently, in the classical limit each theory reduces to its zero mode sector. Furthermore, one easily verifies the classic/quantum correspondence between the two form field theories is a correspondence between the massless sector of one theory and the full spectrum of the another one. In Section 5 we will show that the massless sector of the 2-form theory gives the theory of Kähler gravity on the $C Y_{3}$. Thus the classic/quantum correspondence implies why the massless sector of the 3-form field theory is not the Kodaira-Spencer theory, which is believed to correspond to the topological B string field theory.

\section{Canonical quantization and position/momentum duality}

Now we are able to do canonical quantization. Recall that the actions of 3 and 2-form theories in seven dimensions, respectively, are

$$
\begin{aligned}
& S(\gamma)=\frac{\Lambda}{4 \pi} \int_{S^{1}} d t\{(\dot{\gamma}, \dot{\gamma})+(d \gamma, d \gamma)\}, \\
& S(\omega)=\frac{1}{4 \pi \Lambda} \int_{S^{1}} d t\{(\dot{\omega}, \dot{\omega})+(d \omega, d \omega)\} .
\end{aligned}
$$

The momenta conjugate to $\gamma$ and $\omega$ are, respectively, ${ }^{6}$

$$
\begin{aligned}
P_{\gamma} & =\frac{\Lambda}{2 \pi} \dot{\gamma}, \\
P_{\omega} & =\frac{1}{2 \pi \Lambda} \dot{\omega} .
\end{aligned}
$$

${ }^{6}$ Here we have used the following definition:

$$
\frac{\partial(\psi, \chi)}{\partial \psi}=\frac{\partial(\chi, \psi)}{\partial \psi}=\chi,
$$

which means that the derivation is defined with respect to the product $\wedge *$. 
The Poisson-bracket is given as usual, e.g.,

$$
\left\{\gamma, P_{\gamma}\right\}=1,
$$

which results in

$$
\begin{aligned}
\left\{\gamma_{n}, \gamma_{m}\right\} & =\frac{i}{n} \frac{R}{\Lambda} \delta_{n,-m}, \quad n, m \neq 0, \\
\left\{\gamma_{0}, \gamma_{n}\right\} & =0, \quad n \in \mathbb{Z} .
\end{aligned}
$$

One can define the creation and annihilation operators as usual

$$
\begin{aligned}
a_{n} & =\sqrt{n} \gamma_{n}, \quad n>0, \\
a_{-n}^{\dagger} & =\sqrt{-n} \gamma_{n}, \quad n<0, \\
a_{0} & =a_{0}^{\dagger}=\gamma_{0},
\end{aligned}
$$

satisfying the algebra

$$
\begin{aligned}
{\left[a_{n}, a_{m}^{\dagger}\right] } & =\frac{R}{\Lambda} \delta_{n, m}, \quad n, m \neq 0, \\
{\left[a_{0}, a_{n}\right] } & =\left[a_{0}, a_{n}^{\dagger}\right]=0, \quad n \in \mathbb{Z} .
\end{aligned}
$$

The vacuum state for the 3 -form theory $|0\rangle$ is defined to be the state annihilated by $a_{n}$. There is a subtlety at this point. Since $a_{0}$ commutes with the massive operators $a_{n}^{\dagger}$, one verifies that this operator takes one vacuum state into a nearby equivalent one [11]. Therefore one can define a one-parameter family of vacua such that

$$
\mid \text { vac. }\rangle_{\theta}=\mathrm{e}^{\mathrm{i} \theta a_{0}}|0\rangle \text {. }
$$

We can quantize the 2-form theory (4.2) in the same way and derive the associated vacua family. The difference between these two form field theories is due to the exponent of $\Lambda$ in the commutation algebra. In the 2 -from theory the commutation relation is given by

$$
\begin{aligned}
& {\left[b_{n}, b_{m}^{\dagger}\right]=R \Lambda \delta_{n, m}, \quad n, m \neq 0,} \\
& {\left[b_{0}, b_{n}\right]=\left[b_{0}, b_{n}^{\dagger}\right]=0, \quad n \in \mathbb{Z},}
\end{aligned}
$$

where

$$
\begin{aligned}
b_{n} & =\sqrt{n} \omega_{n}, \quad n>0, \\
b_{-n}^{\dagger} & =\sqrt{-n} \omega_{n}, \quad n<0, \\
b_{o} & =b_{0}^{\dagger}=\omega_{0} .
\end{aligned}
$$


Consequently, the classical limit of one theory $\hbar \rightarrow 0$ (e.g., for 3-form theory it means $\Lambda \rightarrow \infty$ ) corresponds to the quantum extreme of the another theory. This is the classic/quantum correspondence of 2 and 3 -from theories in $C Y_{3}$ induced by the Abelian duality.

\section{$4.1 \quad$ S-duality on $C Y_{3}$}

Now we can understand the duality between the two form gravity theories. The duality is two-edged indeed. Firstly, there is a classic/quantum correspondence between two theories. Secondly, from the classical duality relations $(2.50),(2.51)$ and definition of momentum (4.4), (4.5) we obtain the interesting relations:

$$
\begin{aligned}
& * d \gamma=2 \pi \Lambda P_{\omega}, \\
& * P_{\gamma}=\frac{\Lambda}{2 \pi} d \omega,
\end{aligned}
$$

which can be interpreted as position/momentum or electric/magnetic duality in seven-dimensional form theories [14]. In the six dimensional manifold, this relations in terms of $\mathrm{KK}$ modes reduce to

$$
\begin{aligned}
& * d \gamma_{n}=\mathrm{i} \frac{2 \pi n}{R} \omega_{n}, \\
& * d \omega_{n}=\mathrm{i} \frac{R}{2 \pi n} \gamma_{n} .
\end{aligned}
$$

There is a subtlety in these equations. We see that because of the coefficient $n$, the zero modes of the theories do not play any role in the above duality relations. Zero modes play a significant role in the classical/quantum correspondence. Here we recall our discussion on the classic/quantum correspondence at the end of Section 3. In the classical limit, the 3-form field theory for example, is given by its massless sector, which corresponds to the 2 -form theory in its quantum extreme with both massless and massive excitations.

\section{Theory of Kähler gravity}

Let us see what is the relation between the action

$$
S(\omega) \sim(d \omega, d \omega),
$$

and the theory of Kähler gravity. It is known that the observables of the A-model are elements of $H^{(p, q)}$. For $p=q=1$ one verifies a correspondence between the A-model and the theory of Kähler transformations. 
In physical superstring theories we have $N=(2,2)$ supersymmetry. After the twist to the topological string theories there remain only two supercharges. In the B string field theory the corresponding nilpotent operators are $\partial$ and $\bar{\partial}$. A deformation in the complex structure amounts to deformation of $\bar{\partial}$ given by

$$
\bar{\partial} \rightarrow \bar{\partial}+A,
$$

where $A \in H^{0,1}(T M)$. Requiring that the deformed operator is nilpotent one obtains the Kodaira-Spencer equation [3]

$$
\bar{\partial} A^{\prime}+\frac{1}{2} \partial(A \wedge A)^{\prime}=0,
$$

where $A^{\prime}=A . \Omega$ in which $\Omega$ is the Calabi-Yau 3-form.

In the A model the nilpotent operators are $d$ and $d^{c \dagger} .^{7}$ Operator $d$ is nilpotent without any dependence on the Kähler and/or complex structure. Given a Kähler structure $\omega_{0}$ the $d^{c \dagger}$ operator is defined as follows:

$$
d^{c^{\dagger}}=\left[d, \Lambda_{0}\right],
$$

where $\Lambda_{0}$ is the pull back of multiplication by $\omega_{0}[20]$. As we see, the definition of the operators $d$ and $d^{c \dagger}$ is independent of the complex structure, but for $d^{c \dagger}$ it depends on the Kähler structure as is expected for the A string field theory. By a Kähler transformation one means a deformation of the Kähler form $\omega_{0}$,

$$
\omega_{0} \rightarrow \omega=\omega_{0}+K
$$

where $K$ is a 2 -form, leaving the operator $D^{c \dagger}=d^{c \dagger}+[d, \delta \Lambda]$ be nilpotent. Again $\Lambda=\Lambda_{0}+\delta \Lambda$ is the pull back of the multiplication by $\omega$. Given a complex structure, $\Lambda$ in components is given by the following identities:

$$
\begin{aligned}
\Lambda^{i \bar{j}} \omega_{i \bar{k}} & =\delta_{\bar{k}}^{\bar{j}}, \\
\Lambda^{i \bar{j}} \omega_{k \bar{j}} & =\delta_{k}^{i} .
\end{aligned}
$$

For a general Kähler form $\omega$ it is straightforward to verify that $d^{c \dagger}=[d, \Lambda]$ is nilpotent if and only if

$$
\Lambda_{, k}^{l \bar{j}} \Lambda^{k \bar{m}}=\Lambda_{, k}^{l \bar{m}} \Lambda^{k \bar{j}}
$$

and with the same symmetry for the holomorphic indices. Here by ", $k$ " one means derivation with respect to the holomorphic coordinate $z^{k}$. The

\footnotetext{
${ }^{7}$ By definition, we have$$
d=\partial+\bar{\partial}, \quad d^{c}=\bar{\partial}-\partial .
$$

One can define $d$ without determining complex structure, but to define $d^{\dagger}$ one should choose a complex structure.
} 
identity (5.9) is satisfied iff the inverse of $\Lambda$, i.e., the 2 -form $\omega$ is closed, namely,

$$
\omega_{i \bar{j}, k}=\omega_{k \bar{j}, i}, \quad \omega_{i \bar{j}, \bar{k}}=\omega_{i \bar{k}, \bar{j}} .
$$

Thus the Kähler transformation is given by closed 2-forms $K$ in (5.6). An even dimensional Riemannian manifold can always be equipped with a complex structure for which the action functional

$$
S \sim(d \omega, d \omega)
$$

is positive definite implying the desired condition

$$
d \omega=0
$$

by the classical equation of motion.

In summary the massless sector of the 2-form theory studied in the previous sections corresponds to the A string field theory.

Here we slightly digress to discuss the difference between our method and that of [2] where the theory of Kähler gravity is considered for the first time. The motivation in [2] is to obtain a theory of Kähler transformation to be the mirror of the Kodaira-Spencer theory of deformation of complex structure moduli. The so-called AKS theory of Kähler transformations is given by requiring that the operator

$$
D=d+\left[d^{c \dagger}, K\right]
$$

be nilpotent. On a manifold the operator $d$ can be defined with no reference to the complex structure. Given a Kähler structure by $\omega$ and the corresponding pull back operator $\Lambda$ one can define the operator $d^{c \dagger}$ by equation (5.5) which itself depends on $\omega$. Thus the AKS condition

$$
D^{2}=0
$$

is well defined only by perturbation as far as $d^{c \dagger}$ in equation (5.13) is defined in terms of $d$ and $\omega=\omega_{0}+K$. In our approach one uses the fact that the operator $d$ is defined independent of the Kähler structure and the equation of motion of the A string field theory is obtained from the condition

$$
\left(d^{c \dagger}\right)^{2}=[d, \Lambda]^{2}=0 .
$$

Thus our proposal is equivalent to the AKS conjecture if all terms in the perturbation are considered. 
There are some subtleties in defining the action (5.11). The action $S$ in equation (5.11)

$$
S \sim \int d K \wedge * d K
$$

is defined perturbatively since the Hodge $*$ operation is defined in terms of $\Lambda$, which is known in terms of the deformation $K$ only via the following equation:

$$
\Lambda^{i \bar{j}}=\Lambda_{0}^{i \bar{j}}-\Lambda_{0}^{i \bar{k}} K_{k \bar{k}} \Lambda_{0}^{k \bar{j}}+\cdots
$$

Up to $\mathcal{O}\left(K^{5}\right)$ terms the action is given as follows:

$$
\begin{aligned}
S(K) \sim & \int d V\left(K_{i \bar{j}, \bar{k}} K_{j \bar{i}, k}+K_{j \bar{i}, k} K_{i \bar{j}, \bar{k}}\right) \Lambda^{i \bar{i}}\left(\Lambda^{j \bar{j}} \Lambda^{k \bar{k}}-j \leftrightarrow k\right) \\
= & \int d V\left(K_{i \bar{j}, \bar{k}} K_{j \bar{i}, k}+K_{j \bar{i}, k} K_{i \bar{j}, \bar{k}}\right)\left\{\left(\Lambda_{0}^{i \bar{i}} \Lambda_{0}^{j \bar{j}} \Lambda_{0}^{k \bar{k}}\right)\right. \\
& -\left(\Lambda_{0}^{i \bar{l}} K_{l \bar{l}} \Lambda_{0}^{l \bar{i}} \Lambda_{0}^{j \bar{j}} \Lambda_{0}^{k \bar{k}}+\Lambda_{0}^{i \bar{i}} \Lambda_{0}^{j \bar{l}} K_{l \bar{l}} \Lambda_{0}^{l \bar{l}} \Lambda_{0}^{k \bar{k}}+\Lambda_{0}^{i \bar{i}} \Lambda_{0}^{j \bar{j}} \Lambda_{0}^{k \bar{l}} K_{l \bar{l}} \Lambda_{0}^{l \bar{k}}\right) \\
& +\left(\Lambda_{0}^{i \bar{l}} K_{l \bar{l}} \Lambda_{0}^{l \bar{i}} \Lambda_{0}^{j \bar{m}} K_{m \bar{m}} \Lambda_{0}^{m \bar{j}} \Lambda_{0}^{k \bar{k}}+\Lambda_{0}^{i \bar{l}} K_{l \bar{l}} \Lambda_{0}^{l \bar{i}} \Lambda_{0}^{j \bar{j}} \Lambda_{0}^{k \bar{m}} K_{m \bar{m}} \Lambda_{0}^{m \bar{k}}\right. \\
& +\Lambda_{0}^{i \bar{i}} \Lambda_{0}^{j \bar{l}} K_{l \bar{l}} \Lambda_{0}^{\bar{j}} \Lambda_{0}^{k \bar{m}} K_{m \bar{m}} \Lambda_{0}^{m \bar{k}}+\Lambda_{0}^{i \bar{l}} K_{l \bar{l}} \Lambda_{0}^{l \bar{m}} K_{m \bar{m}} \Lambda_{0}^{m \bar{i}} \Lambda_{0}^{j \bar{j}} \Lambda_{0}^{k \bar{k}} \\
& \left.+\Lambda_{0}^{i \bar{i}} \Lambda_{0}^{i \bar{l}} K_{l \bar{l}} \Lambda_{0}^{l \bar{m}} K_{m \bar{m}} \Lambda_{0}^{m \bar{j}} \Lambda_{0}^{k \bar{k}}+\Lambda_{0}^{i \bar{i}} \Lambda_{0}^{j \bar{j}} \Lambda_{0}^{k \bar{l}} K_{l \bar{l}} \Lambda_{0}^{l \bar{m}} K_{m \bar{m}} \Lambda_{0}^{m \bar{k}}\right) \\
& \left.+\mathcal{O}\left(K^{5}\right)-(j \leftrightarrow k)\right\} .
\end{aligned}
$$

The gauge condition

$$
d^{\dagger} K=0
$$

implies that, e.g.,

$$
\Lambda_{0}^{i \bar{j}} K_{i \bar{l}, \bar{j}}+\mathcal{O}\left(K^{2}\right)=0 .
$$

Thus $K$ in this gauge is given by transverse modes, a well-known notion in the ordinary quantum gravity.

The action (5.16) is appeared to depend on the complex structure though the classical equation $d \omega=0$ was obtained with no reference to the complex structure. For simply connected manifolds, the independence of the action from the complex structure can be verified as follows. Any variation of the complex structure deforms the operator $d$ by $A \in H^{0,1}(T M)$, which is the Kodaira-Spencer field [3]. Since $A . K$ is a $(0,2)$-form, for a simply connected 
manifold it is vanishing identically,

$$
\begin{aligned}
A . K & =(A . K)_{\bar{i} \bar{j}} d \bar{z}^{\bar{i}} \wedge d \bar{z}^{\bar{j}} \\
& =d \eta \\
& =d\left(\eta_{i} d z^{i}+\eta_{\bar{i}} d z^{\bar{i}}\right) \\
& =d\left(\eta_{i} d z^{i}\right)+d\left(\eta_{\bar{i}} d z^{\bar{i}}\right) \\
& =d\left(d\left(\phi^{(1)}\right)\right)+d\left(d\left(\phi^{(2)}\right)\right) \\
& =0
\end{aligned}
$$

where $\eta$ and $\phi^{(i)}$ are 1 and 0 -forms on simply connected manifold, respectively. In the second and fifth lines we used the fact that for a simply connected $C Y_{3}, h^{0,2}=h^{0,1}=0$. Consequently, the theory is classically protected against complex structure deformation. In the next section we study the quantum background independence of this theory.

Before closing this section we address the modification in the action (5.11) for non-simply connected manifolds if it is used to describe the A string filed theory. The A model should be independent of the complex structure moduli. Thus one should add the condition

$$
A \cdot K=0
$$

to the action using the Lagrange multipliers $\alpha_{i}$,

$$
S=(d \omega, d \omega)+\left\{\alpha_{i} \int_{\mathrm{C}_{i}} \text { A.K } K \text { h.c. }\right\},
$$

where $\mathrm{C}_{i}$ 's are $(0,2)$ cycles. The second term induces a coupling between the $\mathrm{A}$ model and $\mathrm{B}+\overline{\mathrm{B}}$ model. ${ }^{8}$ For a Calabi-Yau manifold the constraint (5.22) can be equivalently given by

$$
A^{\prime} \wedge K=0 .
$$

Thus the modified action can be given by

$$
S=(d \omega, d \omega)+\left\{\int_{\mathrm{CY}_{3}} \lambda \wedge A^{\prime} \wedge K+\text { h.c. }\right\}, \quad \lambda \in H^{0,1} .
$$

We postpone further investigation of this theory to future works.

\footnotetext{
${ }^{8}$ In [14], in a different approach to form field theories on $C Y_{3}$, it is argued that on a Calabi-Yau three-fold, the A model is dual to the $\mathrm{B}+\overline{\mathrm{B}}$ model.
} 


\section{Quantum background independence}

The observables of the A string field theory should be independent of the complex structure. In this section at first we construct the necessary tools to study the theory of complex structure deformation, then we use these tools to study the quantum background independence of $H^{2}\left(C Y_{3}\right)$ and $H^{3}\left(C Y_{3}\right)$. The quantum background independence of $H^{3}\left(C Y_{3}\right)$ is worked out previously and is known to result in the holomorphic anomaly equations $[5-7,9]$.

\subsection{The structure of connections}

In this subsection we study the connections corresponding to the deformation of the complex structure on a complex manifolds. It is known that the number of moduli complex structure is $h^{2,1}$. An infinitesimal deformation of complex structure makes the holomorphic differentials $d z^{i}$ on the complex manifold to be mixed with the anti-holomorphic differentials [21]:

$$
\frac{\partial}{\partial t_{M}} d z^{i}=\nu_{j M}^{i} d z^{j}+\mu_{\bar{j} M}^{i} d \bar{z}^{\bar{j}}
$$

where $t_{M}$ are variables of complex structure moduli space and $M$ counts the number of complex structure moduli, $M=1, \ldots, h^{21}$. Fundamental duality relation between basis of forms and basis of vectors,

$$
\begin{aligned}
& \left\langle d z^{i}, \bar{\partial}_{\bar{j}}\right\rangle=\left\langle d \bar{z}^{\bar{i}}, \partial_{j}\right\rangle=0, \\
& \left\langle d z^{i}, \partial_{j}\right\rangle=\delta_{j}^{i}, \\
& \left\langle d \bar{z}^{\bar{i}}, \bar{\partial}_{\bar{j}}\right\rangle=\delta \frac{\bar{i}}{\bar{j}}
\end{aligned}
$$

should be satisfied after the deformation. Therefore, we obtain the following relations for those basis:

$$
\begin{aligned}
& \frac{\partial}{\partial t_{M}} d z^{i}=\nu_{j M}^{i} d z^{j}+\mu_{\bar{j} M}^{i} d \bar{z}^{\bar{j}}, \\
& \frac{\partial}{\partial t_{M}} \partial_{i}=-\nu_{i M}^{j} \partial_{j}+\epsilon_{i M}^{\bar{j}} \bar{\partial}_{\bar{j}}, \\
& \frac{\partial}{\partial t_{M}} d \bar{z}^{\bar{i}}=\xi_{\bar{j} M}^{\bar{i}} d \bar{z}^{\bar{j}}-\epsilon_{j M}^{i} d z^{j} \\
& \frac{\partial}{\partial t_{M}} \bar{\partial}_{\bar{i}}=-\xi_{\bar{i} M}^{\bar{j}} \bar{\partial}_{\bar{j}}-\mu_{\bar{i} M}^{\bar{j}} \bar{\partial}_{\bar{j}} .
\end{aligned}
$$


Thus we have four sets of independent connections $\mu_{M}, \nu_{M}, \epsilon_{M}$, and $\xi_{M}$ of complex structure deformation on a complex manifold. In the next subsection we show on a $C Y_{3}$ manifold only two sets of these connections remain and two other sets are vanishing identically.

\subsection{Complex structure independence in form field theories}

In the 3-form gravity theory, which is defined on a Calabi-Yau three-fold manifold, real closed 3-form $\gamma_{0}$ can be decomposed on the basis consisting of the Calabi-Yau 3-form $\Omega \in H^{3,0},{ }^{9}$ its covariant derivatives with respect to the moduli directions $t_{M}, \nabla_{M} \Omega \in H^{2,1},{ }^{10}$ and their complex conjugates,

$$
\gamma_{0}=\lambda^{-1} \Omega+x^{M} \nabla_{M} \Omega+\bar{x}^{\bar{M}} \bar{\nabla}_{\bar{M}} \bar{\Omega}+\bar{\lambda}^{-1} \bar{\Omega} .
$$

Locally the Calabi-Yau 3-form takes the form,

$$
\Omega=\frac{1}{3 !} h(z) \varepsilon_{i j k} d z^{i} \wedge d z^{j} \wedge d z^{k}
$$

and

$$
\frac{\partial}{\partial t_{M}} \Omega=\frac{1}{3 !} h(z)_{, M} \varepsilon_{i j k} d z^{i} \wedge d z^{j} \wedge d z^{k}+\frac{1}{2 !} h(z) \varepsilon_{i j k} \frac{\partial}{\partial t_{M}}\left(d z^{i}\right) \wedge d z^{j} \wedge d z^{k} .
$$

By expanding this in terms of $\mu_{\bar{j} M}^{i}$ and $\nu_{j M}^{i}$ and using equations (6.3) one verifies that $\mu_{j M}^{i}$ and $\nu_{j M}^{i}$ are generators of bidegree $(2,1)$ and $(3,0)$-forms, respectively.

Since on a Calabi-Yau three-fold $\partial_{M} \bar{\Omega}=0$ and $\Omega$ is a nowhere vanishing holomorphic 3 -form, by expanding $\partial_{M} \bar{\Omega}$ one can verify that $\xi_{\bar{b} M}^{\bar{a}}=0=\epsilon_{b M}^{a}$

${ }^{9}$ For Calabi-Yau three-fold we have $h^{0,0}=h^{3,0}=h^{0,3}=h^{3,3}=1$.

${ }^{10} H^{3}\left(C Y_{3}\right)$ forms a bundle over the moduli space of the complex structures of the $C Y_{3}$. The holomorphic 3 -form defines a line sub-bundle $\mathcal{L}$ and on this sub-bundle one can define a connection $\nabla_{M}=\partial_{M}+\partial_{M} K$ where $K$ is the Kähler potential defined as follows:

$$
K=-\ln i \int \bar{\Omega} \wedge \Omega
$$


and equations (6.3) simplify to

$$
\begin{aligned}
\frac{\partial}{\partial t_{M}} d z^{i} & =\nu_{j M}^{i} d z^{j}+\mu_{j M}^{i} d \bar{z}^{j}, \\
\frac{\partial}{\partial t_{M}} \partial_{i} & =-\nu_{i M}^{j} \partial_{j}, \\
\frac{\partial}{\partial t_{M}} d \bar{z}^{i} & =0 \\
\frac{\partial}{\partial t_{M}} \bar{\partial}_{\bar{i}} & =-\mu_{\bar{i} M}^{\bar{j}} \bar{\partial}_{\bar{j}} .
\end{aligned}
$$

Consequently, all of the complex structure deformation on Calabi-Yau threefold are given by $\mu_{\bar{b} M}^{a}$ and $\nu_{b M}^{a}$.

In 3 -form theory it is shown that the holomorphic anomaly equations depend only on the $\mu_{\bar{b} M}^{a}$ connections [4], which can be equivalently obtained by imposing the background independence condition on $H^{3}\left(C Y_{3}\right)[6,9]$.

For the 2-form gravity theory one deals with a 2 -form $\omega_{0}$ on the $C Y_{3}$. The 2-form $\omega_{0}$ is given in terms of a basis of $H^{2}\left(C Y_{3}\right)$ as follows:

$$
\omega_{0}=x_{I} \omega^{I}(z),
$$

where $I$ runs over the basis of $H^{2}\left(C Y_{3}\right)$. If a compact simply connected $\mathrm{CY}_{3}$ manifold is considered, since for any suitable complex structure we have $h^{2,0}=0$ any basis for $H^{2}\left(C Y_{3}\right)$ is a basis for the Kähler forms. For a given complex structure $\omega^{I}(z)=\omega^{I}(z)_{a \bar{b}} d z^{a} \wedge d \bar{z}^{\bar{b}}$ for $I=1, \cdots h^{1,1}$. Thus one obtains

$$
\partial_{M} \omega^{I}=\left(\omega_{i \bar{j}}^{I}\right)_{, M} d z^{i} \wedge d \bar{z}^{\bar{j}}+x_{I} \omega_{i \bar{j}}^{I} \partial_{M}\left(d z^{i}\right) \wedge d \bar{z}^{\bar{j}} .
$$

By expanding this formula in terms of $\mu_{j M}^{i}$ and $\nu_{j M}^{i}$ and noting that $h^{2,0}$ $\left(C Y_{3}\right)=0$ one obtains that $\mu_{j}^{i}$ is a null operator on the $H^{2}$, i.e.,

$$
\mu_{\bar{i} M}^{i} \omega_{i \bar{j}} d \bar{z}^{\bar{i}} \wedge d \bar{z}^{\bar{j}}=0 .
$$

By imposing the background (i.e., complex structure) independence condition on the symplectic 2 -form $\omega_{0}$, one obtains

$$
\left[\nu_{M i}^{j} \omega_{j \bar{i}}^{I}+\left(\omega_{i \bar{j}}^{I}\right)_{M}\right] d z^{i} \wedge d \bar{z}^{\bar{j}}=0 .
$$

Thus, $\nu_{j M}^{i}$ are generators of transformation along the complex structure moduli directions for $\mathrm{H}^{2}\left(\mathrm{CY}_{3}\right)$. 


\section{Appendices}

\section{A Abelian duality in seven dimensions}

At the end of Section 2 we saw that a coefficient multiplies the partition functions of 2-form theory in seven dimensions. In this appendix we show that this coefficient is a topological invariant, which only depends on the Hodge numbers of $C Y_{3}$. For deriving that coefficient one should calculate two terms: the Gaussian integral over $A^{\prime}, \int \mathcal{D} A^{\prime} \mathrm{e}^{-\frac{\Lambda}{4 \pi} \int_{\Sigma} A^{\prime} \wedge_{*_{7}} A^{\prime}}$, and the grassmanian integral over $c$ and $\bar{c}, \int \mathcal{D} \bar{c} \mathcal{D} c \mathrm{e}^{-\frac{\Lambda}{4 \pi} \int_{\Sigma} \bar{c} c}$. For the first one, we note that $A^{\prime}$ is a 4 -form and the Hodge star operator is a linear map. Thus this term gives a factor of $\int d y \mathrm{e}^{-(\Lambda / 4 \pi) y}=\sqrt{\frac{\pi}{\Lambda / 4 \pi}}$ for each 4-form. ${ }^{11}$

For the second term it should be noted that the path integral over the ghost fields run over the set of grassmanian $r$-forms and $(7-r)$-forms where $r=0, \ldots, 7$ (note that either the $r$ or $(7-r)$-form is an even form). For each set of ghost fields one obtains $\int \mathcal{D} \bar{c} \mathcal{D} c \mathrm{e}^{-\frac{\Lambda}{4 \pi} \int \bar{c} c}=\Lambda / 4 \pi$. Thus the overall factor is

$$
\mathcal{C}(\Sigma)=(2 \pi)^{1+h^{2,1}+h^{1,1} / 2}(\Lambda / 2 \pi)^{4+h^{2,1}+3 h^{1,1} / 2},
$$

where we have used the following identities for the Hodge numbers of $\Sigma=$ $C Y_{3} \times S^{1}[18]:$

$$
\begin{aligned}
& b^{0}(\Sigma)=b^{7}(\Sigma)=1, \\
& b^{1}(\Sigma)=b^{6}(\Sigma)=2, \\
& b^{2}(\Sigma)=b^{5}(\Sigma)=h^{1,1}\left(C Y_{3}\right), \\
& b^{3}(\Sigma)=b^{4}(\Sigma)=2 h^{2,1}\left(C Y_{3}\right)+h^{1,1}\left(C Y_{3}\right)+2 .
\end{aligned}
$$

\section{B Relation between the operators $d$ and $*$ in $m$ and $m+1$ dimensions}

In this appendix we deal with some relations between the operators in $m$ and $m+1$ dimensions. In Section 2 we used two elementary operators, namely the exterior derivative $d$ and the Hodge star $*$ in six and seven dimensions.

\footnotetext{
${ }^{11} y$ is a normalization real variable and is defined such that $\int \mathcal{D} A^{\prime} \mathrm{e}^{-\int_{7} A^{\prime} \wedge * A^{\prime}}=\int d y \mathrm{e}^{-y}$ $[22]$.
} 
Operator $d$ is a map $d: \Omega^{r}(M) \rightarrow \Omega^{r+1}(M)$, where $\Omega^{r}(M)$ are r-forms of manifold $M$, whose action on a $r$-form

$$
\eta_{r}=\frac{1}{r !} \eta_{\mu_{1} \cdots \mu_{r}} d x^{\mu_{1}} \wedge \cdots \wedge d x^{\mu_{r}}
$$

is defined by

$$
d \eta_{r}=\frac{1}{r !}\left(\frac{\partial}{\partial x^{\nu}} \eta_{\mu_{1} \cdots \mu_{r}}\right) d x^{\nu} \wedge d x^{\mu_{1}} \wedge \cdots \wedge d x^{\mu_{r}}
$$

To obtain the relation between exterior derivative in $m$ and $(m+1)$ dimensional manifolds, one should note that for our purpose $(m+1)$ th direction in $(m+1)$-dimensional manifold is perpendicular to other directions. Therefore it is obvious that,

$$
d_{m+1}=d_{m}+d t \wedge \frac{\partial}{\partial t}
$$

where subscripts $m$ and $m+1$ indicate the dimension of manifold and $t$ is the coordinate of $(m+1)$ th direction.

For Hodge star operator there are some subtleties. Operator $*$ is a linear map $\Omega^{r}(M) \rightarrow \Omega^{m-r}(M)$, where $m$ is the dimension of manifold $M$. The operation of the Hodge $*$ on an element of $\Omega^{r}(M)$ is defined by

$$
\begin{aligned}
& *\left(d x^{\mu_{1}} \wedge \cdots \wedge d x^{\mu_{r}}\right) \\
& \quad=\frac{\sqrt{|g|}}{r !(m-r) !} g^{\mu_{1} \nu_{1}} \cdots g^{\mu_{r} \nu_{r}} \varepsilon_{\nu_{1} \cdots \nu_{r} \nu_{r+1} \cdots \nu_{m}} d x^{\nu_{r+1}} \wedge \cdots \wedge d x^{\nu_{m}}
\end{aligned}
$$

in which $g^{\mu \nu}$ is the metric of manifold $M, g$ is the determinant of $g_{\mu \nu}$, and $\mu_{i}, \nu_{i}=1, \ldots, m$.

We consider two independent cases: an $r$-form in $m+1$ dimensions which is an $r$-form along $m$-dimensional manifold, and an $r$-form in $m+1$ dimensions which is an $(r-1)$-form along $m$-dimensional manifold and an 1 -form along the $(m+1)$ th direction. For the first case $\eta_{r}=\frac{1}{r !} \eta_{\mu_{1} \cdots \mu_{r}} d x^{\mu_{1}} \wedge \cdots \wedge$ $d x^{\mu_{r}}$, thus,

$$
*_{m} \eta_{r}=\frac{\sqrt{|g|}}{r !(m-r) !} \eta_{\mu_{1} \cdots \mu_{r}} g^{\mu_{1} \nu_{1}} \cdots g^{\mu_{r} \nu_{r}} \varepsilon_{\nu_{1} \cdots \nu_{r} \nu_{r+1} \cdots \nu_{m}} d x^{\nu_{r+1}} \wedge \cdots \wedge d x^{\nu_{m}}
$$




$$
\begin{aligned}
*_{m+1} \eta_{r}= & \frac{\sqrt{\left|g^{\prime}\right|}}{r !(m+1-r) !} \eta_{\mu_{1} \cdots \mu_{r}} g^{\mu_{1} \nu_{1}} \cdots g^{\mu_{r} \nu_{r}} \varepsilon_{\nu_{1} \cdots \nu_{r} \nu_{r+1} \cdots \nu_{m} \nu_{m+1}} \\
& \times d x^{\nu_{r+1}} \wedge \cdots \wedge d x^{\nu_{m}} \wedge d x^{\nu_{m+1}}
\end{aligned}
$$

where $g$ and $g^{\prime}$ are determinant of metrics in $m$ and $(m+1)$-dimensional manifolds, respectively, $\varepsilon_{1 \cdots m}$ is the totally antisymmetric tensor, $\mu_{i}=1, \ldots$, $m, \nu_{i}=1, \ldots, m$ for the first equation, and $\nu_{i}=1, \ldots, m$ for the second equation. Since the $(m+1)$ th direction is perpendicular to the other directions one obtains $g^{\mu_{i}, m+1}=\delta_{\mu_{i}, m+1}$ and $g=g^{\prime}$. Therefore in equation (B.6) $\nu_{i}$ only takes value in the range $1 \leq \nu_{i} \leq m$ for $i=1, \ldots, r+1$. Since there are $(m+1-r)$ positions for $\nu_{m+1}$ in of $\varepsilon_{1 \cdots m}$, one obtains

$$
\begin{aligned}
*_{m+1} \eta_{r}= & \frac{\sqrt{|g|}}{r !(m-r) !} \eta_{\mu_{1} \cdots \mu_{r}} g^{\mu_{1} \nu_{1}} \cdots g^{\mu_{r} \nu_{r}} \varepsilon_{\nu_{1} \cdots \nu_{r} \nu_{r+1} \cdots \nu_{m} m+1} \\
& \times d x^{\nu_{r+1}} \wedge \cdots \wedge d x^{\nu_{m}} \wedge d x^{m+1}
\end{aligned}
$$

where $\mu_{i}, \nu_{i}=1 \cdots m$. By comparing equation (B.5) with equation (B.7) one obtains the following relation:

$$
*_{m+1} \eta_{r}=*_{m} \eta_{r} \wedge d x^{m+1} .
$$

In the second case, we assume,

$$
\begin{aligned}
\eta_{r+1} & =\frac{1}{(r+1) !} \eta_{\mu_{1} \cdots \mu_{r} m+1} d x^{\mu_{1}} \wedge \cdots \wedge d x^{\mu_{r}} \wedge d x^{m+1} \\
& =\frac{1}{r+1} \eta_{r m+1}^{\prime} \wedge d x^{m+1}
\end{aligned}
$$

where $\eta_{r m+1}^{\prime} \equiv \frac{1}{r !} \eta_{\mu_{1} \cdots \mu_{r} m+1} d x^{\mu_{1}} \wedge \cdots \wedge d x^{\mu_{r}}$ is an $(r+1)$-form along the $(m+1)$-dimensional manifold and an $r$-form along the $m$-dimensional manifold. Hodge star operation in the $m$-manifold gives

$$
\begin{aligned}
*_{m} \eta_{r+1}= & \frac{1}{r+1} *_{m}\left(\eta_{r m+1}^{\prime}\right) \wedge d x^{m+1} \\
= & \frac{1}{r+1} \frac{\sqrt{|g|}}{r !(m-r) !} \eta_{\mu_{1} \cdots \mu_{r} m+1} g^{\mu_{1} \nu_{1}} \cdots g^{\mu_{r} \nu_{r}} \varepsilon_{\nu_{1} \cdots \nu_{r} \nu_{r+1} \cdots \nu_{m}} \\
& \times d x^{\nu_{r+1}} \wedge \cdots \wedge d x^{\nu_{m}} \wedge d x^{m+1},
\end{aligned}
$$


where $\mu_{i}, \nu_{i}=1 \cdots m$. In addition

$$
\begin{aligned}
*_{m+1} & \eta_{r+1} \\
= & \frac{\sqrt{\left|g^{\prime}\right|}}{(r+1) !(m-r) !} \eta_{\mu_{1} \cdots \mu_{r} m+1} \\
& \times g^{\mu_{1} \nu_{1}} \cdots g^{\mu_{r} \nu_{r}} g^{m+1 \nu} \varepsilon_{\nu_{1} \cdots \nu_{r} \nu \nu_{r+2} \cdots \nu_{m+1}} d x^{\nu_{r+2}} \wedge \cdots \wedge d x^{\nu_{m+1}} \\
= & \frac{\sqrt{|g|}}{(r+1) !(m-r) !} \eta_{\mu_{1} \cdots \mu_{r} m+1} \\
& \times g^{\mu_{1} \nu_{1}} \cdots g^{\mu_{r} \nu_{r}} g^{m+1, m+1} \varepsilon_{\nu_{1} \cdots \nu_{r}, m+1, \nu_{r+2} \cdots \nu_{m+1}} d x^{\nu_{r+2}} \wedge \cdots \wedge d x^{\nu_{m+1}} \\
= & (-)^{m-r} \frac{\sqrt{|g|}}{(r+1) !(m-r) !} \eta_{\mu_{1} \cdots \mu_{r} m+1} \\
& \times g^{\mu_{1} \nu_{1}} \cdots g^{\mu_{r} \nu_{r}} \varepsilon_{\nu_{1} \cdots \nu_{r} \nu_{r+2} \cdots \nu_{m+1} m+1} d x^{\nu_{r+2}} \wedge \cdots \wedge d x^{\nu_{m+1}} .
\end{aligned}
$$

Comparing equation (B.10) with equation (B.11) one verifies that

$$
\left(*_{m+1} \eta_{r+1}\right) \wedge d x^{m+1}=(-)^{(m-r)} *_{m} \eta_{r+1} .
$$

If the signature of $(m+1)$ th direction is Lorentzian, then $g^{\mu_{i}, m+1}=-\delta_{\mu_{i}, m+1}$ and one should consider an extra $(-1)$ in the above equation.

As an application of these relations one can show that for an $r$-form $\eta_{r}$ along six dimensions of original seven-dimensional manifold

$$
\begin{aligned}
d_{7}^{\dagger} d_{7} \eta_{r} & =(-)^{7(r+2)+1} *_{7} d_{7} *_{7} d_{7} \eta_{r} \\
& =(-)^{r+1} *_{7} d_{7} *_{7}\left(d_{6} \eta_{r}+d t \wedge \dot{\eta}_{r}\right) \\
& =(-)^{r+1} *_{7} d_{7}\left(*_{6} d_{6} \eta_{r} \wedge d t+*_{6} \dot{\eta}_{r}\right) \\
& =(-)^{r+1} *_{7}\left(d_{6} *_{6} d_{6} \eta_{r} \wedge d t+d_{6} *_{6} \dot{\eta}_{r}+d t \wedge *_{6} \ddot{\eta}_{r}\right) \\
& =(-)^{r+1}\left((-)^{r} *_{6} d_{6} *_{6} d_{6} \eta_{r}+*_{6} d_{6} *_{6} \dot{\eta}_{r} \wedge d t+*_{6} *_{6} \ddot{\eta}_{r}\right) \\
& =d_{6}^{\dagger} d_{6} \eta_{r}-\ddot{\eta}_{r}+(-)^{r} d_{6}^{\dagger} \dot{\eta}_{r} \wedge d t,
\end{aligned}
$$

where equation (2.1), (2.2) are used to this derivation. Also for $\eta_{r+1}=$ $\eta_{r} \wedge d t$, one obtains

$$
\begin{aligned}
d_{7}^{\dagger} d_{7}\left(\eta_{r} \wedge d t\right) & =(-)^{7(r+2)+1} *_{7} d_{7} *_{7} d_{7}\left(\eta_{r} \wedge d t\right) \\
& =(-)^{r+1} *_{7} d_{7} *_{7}\left(d_{6} \eta_{r} \wedge d t\right) \\
& =*_{7} d_{7}\left(*_{6} d_{6} \eta_{r}\right) \\
& =*_{7}\left(d_{6} *_{6} d_{6} \eta_{r}+d t \wedge *_{6} d_{6} \dot{\eta}_{r}\right) \\
& =*_{6} d_{6} *_{6} d_{6} \eta_{r} \wedge d t+*_{6} *_{6} d_{6} \dot{\eta}_{r} \\
& =-d_{6}^{\dagger} d_{6} \eta_{r} \wedge d t+(-)^{r+1} d_{6} \dot{\eta}_{r}
\end{aligned}
$$




\section{References}

[1] E. Witten, Chern-Simons gauge theory as a string theory, Prog. Math. 133 (1995), 637, [hep-th/9207094].

[2] M. Bershadsky and V. Sadov, Theory of Kähler Gravity, Int. J. Mod. Phys. A11 (1996), 4689-4730, [hep-th/9410011].

[3] M. Bershadsky, S. Cecotti, H. Ooguri and C. Vafa, Kodaira-Spencer theory of gravity and exact results for quantum string amplitudes, Commun. Math. Phys. 165 (1994), 311, [hep-th/9309140].

[4] M. Bershadsky, S. Cecotti, H. Ooguri and C. Vafa, Holomorphic anomalies in topological field theories, Nucl. Phys. B405 (1993), 279, [hep-th/9302103].

[5] E. Witten, Quantum background independence in string theory, hep-th/9306122.

[6] E. Verlinde, Attractors and the Holomorphic anomaly, hep-th/0412139.

[7] A. A. Gerasimov and S. L. Shatashvili, Towards integrability of topological strings I: three-forms on Calabi-Yau manifolds, JHEP 0411 (2004), 074, [hep-th/0409238].

[8] S. Cecotti, and C. Vafa, Topological antitopological fusion, Nucl. Phys. B367 (1991), 359.

[9] F. Loran, Kähler Quantization of $H 3(C Y 3, R)$ and the holomorphic anomaly, JHEP 0512 (2005), 004, [hep-th/0510163].

[10] C. Vafa, Superstrings and topological strings at large N, J. Math. Phys. 42 (2001), 2798, [hep-th/0008142].

[11] P. Candelas and X. Ossa, Moduli space of Calabi-Yau Manifolds, Nucl.Phys. B355 (1991), 455.

[12] K. Hori, S. Katz, A. Klemm, R. Pandharipande, R. P. Thomas, C. Vafa, R. Vakil, and E. Zaslow, Mirror symmetry, vol. 1 of Clay Mathematics Monographs, American Mathematical Society, Providence, RI, 2003, ISBN: 0821829556.

[13] N. Hitchin, Stable forms and special metrics, math.DG/0107101.

[14] R. Dijkgraaf, S. Gukov, A. Neitzke, and C. Vafa, Topological M-theory as unification of form theories of gravity, Adv. Theor. Math. Phys. 9 (2005), 593-602, [hepth/0411073].

[15] N. Nekrasov, H. Ooguri and C. Vafa, S-duality and topological strings, JHEP 0410 (2004), 009, [hepth/0403167].

[16] A. Neitzke and C. Vafa, $N=2$ strings and the twistorial Calabi-Yau [hep-th/0402128]. 
[17] E. M. Prodanov and S. Sen, Abelian duality, Phys. Rev. D62 (2000), 045009, [hep-th/9906143].

[18] M. Nakahara, Geometry, topology and physics, A. Hilger, Bristol, England, 1990, ISBN: 0852740948.

[19] E. Witten, Abelian duality, in Quantum Fields and Strings: A Course for Mathematicians, eds. P. Deligne et al. vol. 2, American Mathematical Society, 1999, ISBN-13: 978-0-8218-2014-8.

[20] P. Griffiths and J. Harris, Principles of algebraic geometry, Wiley, New York, Providence, RI, 1978, ISBN: 0471327921.

[21] A. Strominger, Special geometry, Comm. Math. Phys. 133 (1990), 163.

[22] B. Hatfield, Quantum field theory of point particles and strings, Addison-Wesley, Reading, MA, 1992, ISBN: 0201360799. 